\title{
Is there a computable upper bound on the heights of rational solutions of a Diophantine equation with a finite number of solutions?
}

\author{
Krzysztof Molenda, Agnieszka Peszek, Maciej Sporysz, Apoloniusz Tyszka \\ University of Agriculture \\ Faculty of Production and Power Engineering \\ Balicka 116B, 30-149 Kraków, Poland \\ Email: \{Krzysztof.Molenda, Agnieszka.Peszek, Maciej.Sporysz\}@urk.edu.pl, rttyszka@cyf-kr.edu.pl
}

\begin{abstract}
The height of a rational number $\frac{p}{q}$ is denoted by $h\left(\frac{p}{q}\right)$ and equals $\max (|p|,|q|)$ provided $\frac{p}{q}$ is written in lowest terms. The height of a rational tuple $\left(x_{1}, \ldots, x_{n}\right)$ is denoted by $h\left(x_{1}, \ldots, x_{n}\right)$ and equals $\max \left(h\left(x_{1}\right), \ldots, h\left(x_{n}\right)\right)$. Let $G_{n}=\left\{x_{i}+1=x_{k}: i, k \in\{1, \ldots, n\}\right\} \cup\left\{x_{i} \cdot x_{j}=x_{k}: i, j, k \in\{1, \ldots, n\}\right\}$. Let $f(1)=1$, and let $f(n+1)=2^{2} f(n)$ for every positive integer $n$. We conjecture: (1) if a system $\mathcal{S} \subseteq G_{n}$ has only finitely many solutions in rationals $x_{1}, \ldots, x_{n}$, then each such solution $\left(x_{1}, \ldots, x_{n}\right)$ satisfies $h\left(x_{1}, \ldots, x_{n}\right) \leqslant\left\{\begin{array}{ll}1 & \text { (if } n=1) \\ 2^{2^{n-2}} & \text { (if } n \geqslant 2)\end{array} ;\right.$ (2) if a system $\mathcal{S} \subseteq G_{n}$ has only finitely many solutions in non-negative rationals $x_{1}, \ldots, x_{n}$, then each such solution $\left(x_{1}, \ldots, x_{n}\right)$ satisfies $h\left(x_{1}, \ldots, x_{n}\right) \leqslant f(2 n)$. We prove: (1) both conjectures imply that there exists an algorithm which takes as input a Diophantine equation, returns an integer, and this integer is greater than the heights of rational solutions, if the solution set is finite; (2) both conjectures imply that the question whether or not a given Diophantine equation has only finitely many rational solutions is decidable by a single query to an oracle that decides whether or not a given Diophantine equation has a rational solution.
\end{abstract}

Index Terms-Diophantine equation which has only finitely many rational solutions, Hilbert's Tenth Problem for $\mathbb{Q}$, relative decidability, upper bound on the heights of rational solutions.

\section{Introduction}

$\mathbf{T}$ HE height of a rational number $\frac{p}{q}$ is denoted by $h\left(\frac{p}{q}\right)$ and equals $\max (|p|,|q|)$ provided $\frac{p}{q}$ is written in lowest terms. The height of a rational tuple $\left(x_{1}, \ldots, x_{n}\right)$ is denoted by $h\left(x_{1}, \ldots, x_{n}\right)$ and equals $\max \left(h\left(x_{1}\right), \ldots, h\left(x_{n}\right)\right)$. We attempt to formulate a conjecture which implies a positive answer to the following open problem:

Is there an algorithm which takes as input a Diophantine equation, returns an integer, and this integer is greater than the heights of rational solutions, if the solution set is finite?

\section{Conjecture 1 and its equivalent form}

Observation 1. Only $x_{1}=0$ and $x_{1}=1$ solve the equation $x_{1} \cdot x_{1}=x_{1}$ in integers (rationals, real numbers, complex num- bers). For each integer $n \geqslant 2$, the following system

$$
\left\{\begin{aligned}
x_{1} \cdot x_{1} & =x_{1} \\
x_{1}+1 & =x_{2} \\
x_{1} \cdot x_{2} & =x_{2} \\
\forall i \in\{2, \ldots, n-1\} x_{i} \cdot x_{i} & =x_{i+1}(\text { if } n \geqslant 3)
\end{aligned}\right.
$$

has exactly one integer (rational, real, complex) solution, namely $\left(1,2,4,16,256, \ldots, 2^{2^{n-3}}, 2^{2^{n-2}}\right)$.

Let

$$
\begin{gathered}
G_{n}=\left\{x_{i}+1=x_{k}: i, k \in\{1, \ldots, n\}\right\} \cup \\
\left\{x_{i} \cdot x_{j}=x_{k}: i, j, k \in\{1, \ldots, n\}\right\}
\end{gathered}
$$

Conjecture 1. If a system $\mathcal{S} \subseteq G_{n}$ has only finitely many solutions in rationals $x_{1}, \ldots, x_{n}$, then each such solution $\left(x_{1}, \ldots, x_{n}\right)$ satisfies

$$
h\left(x_{1}, \ldots, x_{n}\right) \leqslant \begin{cases}1 & (\text { if } n=1) \\ 2^{2^{n-2}} & (\text { if } n \geqslant 2)\end{cases}
$$

Observation 1 implies that the bound

$$
\begin{cases}1 & \text { (if } n=1) \\ 2^{2^{n-2}} & \text { (if } n \geqslant 2)\end{cases}
$$

cannot be decreased.

Conjecture 1 is equivalent to the following conjecture on rational arithmetic: if rational numbers $x_{1}, \ldots, x_{n}$ satisfy

$$
h\left(x_{1}, \ldots, x_{n}\right)> \begin{cases}1 & (\text { if } n=1) \\ 2^{2^{n-2}} & (\text { if } n \geqslant 2)\end{cases}
$$

then there exist rational numbers $y_{1}, \ldots, y_{n}$ such that

$$
h\left(x_{1}, \ldots, x_{n}\right)<h\left(y_{1}, \ldots, y_{n}\right)
$$

and for every $i, j, k \in\{1, \ldots, n\}$

$$
\left(x_{i}+1=x_{k} \Longrightarrow y_{i}+1=y_{k}\right) \wedge\left(x_{i} \cdot x_{j}=x_{k} \Longrightarrow y_{i} \cdot y_{j}=y_{k}\right)
$$

Theorem 1. Conjecture 1 is true if and only if the execution of Flowchart 1 prints infinitely many numbers. 


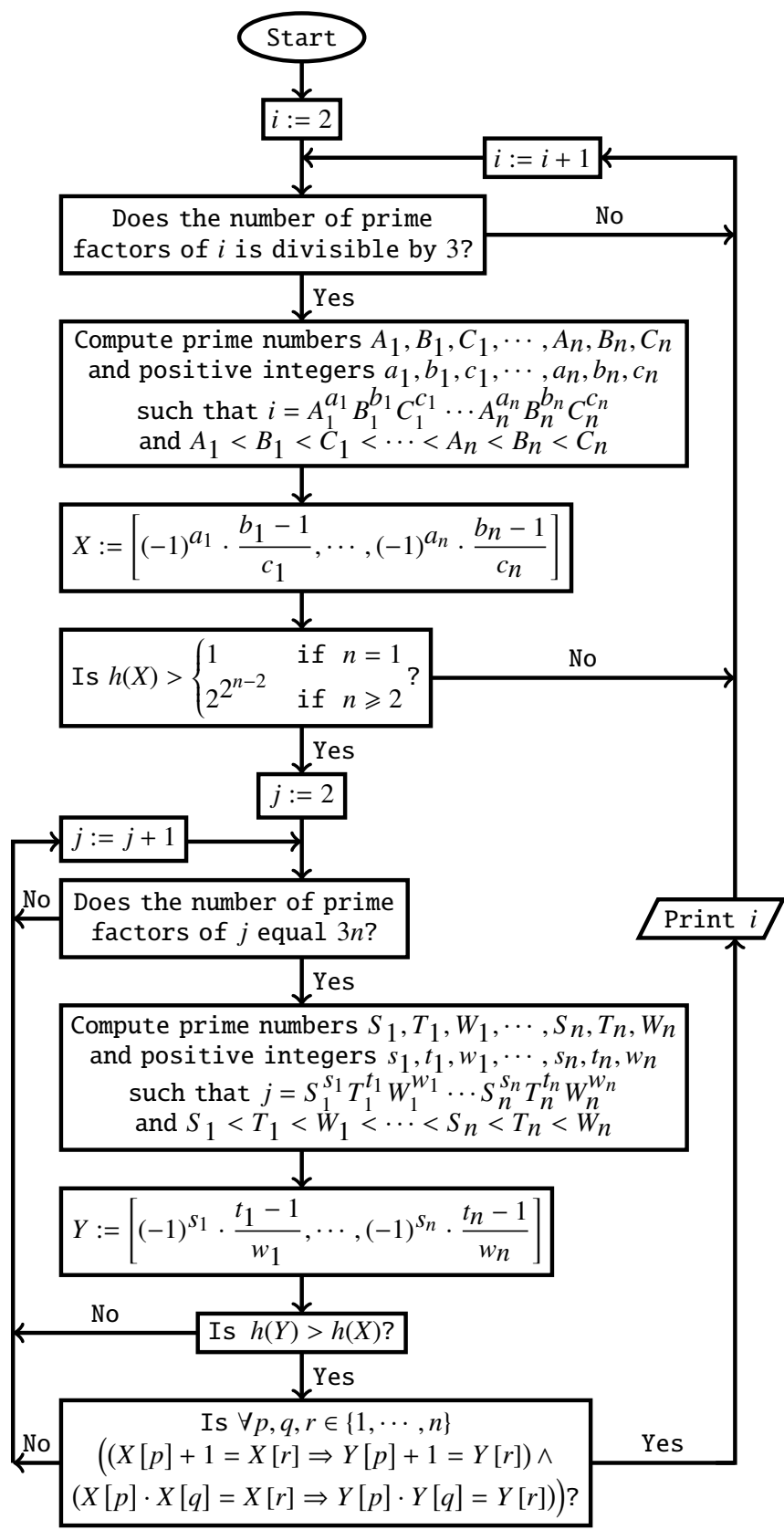

Flowchart 1: An infinite-time computation which decides whether or not Conjecture 1 is true

Proof. Let $\Gamma_{3}$ denote the set of all integers $i \geqslant 2$ whose number of prime factors is divisible by 3 . The claimed equivalence is true because the algorithm from Flowchart 1 applies a surjective function $\eta: \Gamma_{3} \rightarrow \bigcup_{n=1}^{\infty} \mathbb{Q}^{n}$.

Corollary 1. Conjecture 1 can be written in the form $\forall x \in \mathbb{N} \exists y \in \mathbb{N} \phi(x, y)$, where $\phi(x, y)$ is a computable predicate.

\section{Algebraic lemmas - part 1}

Let $\mathcal{R}$ denote the class of all rings, and let $\mathcal{R} n g$ denote the class of all rings $\boldsymbol{K}$ that extend $\mathbb{Z}$. Let

$$
\begin{gathered}
E_{n}=\left\{1=x_{k}: k \in\{1, \ldots, n\}\right\} \cup \\
\left\{x_{i}+x_{j}=x_{k}: i, j, k \in\{1, \ldots, n\}\right\} \cup \\
\left\{x_{i} \cdot x_{j}=x_{k}: i, j, k \in\{1, \ldots, n\}\right\}
\end{gathered}
$$

Lemma 1. ([12, p. 720]) Let $D\left(x_{1}, \ldots, x_{p}\right) \in \mathbb{Z}\left[x_{1}, \ldots, x_{p}\right]$. Assume that $d_{i}=\operatorname{deg}\left(D, x_{i}\right) \geqslant 1$ for each $i \in\{1, \ldots, p\}$. We can compute a positive integer $n>p$ and a system $T \subseteq E_{n}$ which satisfies the following three conditions:

Condition 1. If $\boldsymbol{K} \in \mathcal{R} n g \cup\{\mathbb{N}, \mathbb{N} \backslash\{0\}\}$, then

$$
\begin{gathered}
\forall \tilde{x}_{1}, \ldots, \tilde{x}_{p} \in \boldsymbol{K}\left(D\left(\tilde{x}_{1}, \ldots, \tilde{x}_{p}\right)=0 \Longleftrightarrow\right. \\
\left.\exists \tilde{x}_{p+1}, \ldots, \tilde{x}_{n} \in \boldsymbol{K}\left(\tilde{x}_{1}, \ldots, \tilde{x}_{p}, \tilde{x}_{p+1}, \ldots, \tilde{x}_{n}\right) \text { solves } T\right)
\end{gathered}
$$

Condition 2. If $\boldsymbol{K} \in \mathcal{R} n g \cup\{\mathbb{N}, \mathbb{N} \backslash\{0\}\}$, then for each $\tilde{x}_{1}, \ldots, \tilde{x}_{p} \in \boldsymbol{K}$ with $D\left(\tilde{x}_{1}, \ldots, \tilde{x}_{p}\right)=0$, there exists a unique tuple $\left(\tilde{x}_{p+1}, \ldots, \tilde{x}_{n}\right) \in \boldsymbol{K}^{n-p}$ such that the tuple $\left(\tilde{x}_{1}, \ldots, \tilde{x}_{p}, \tilde{x}_{p+1}, \ldots, \tilde{x}_{n}\right)$ solves $T$.

Condition 3. If $M$ denotes the maximum of the absolute values of the coefficients of $D\left(x_{1}, \ldots, x_{p}\right)$, then

$$
n=(M+2)^{\left(d_{1}+1\right) \cdot \ldots \cdot\left(d_{p}+1\right)}-1
$$

Conditions 1 and 2 imply that for each $\boldsymbol{K} \in \mathcal{R} n \cup\{\mathbb{N}, \mathbb{N} \backslash\{0\}\}$, the equation $D\left(x_{1}, \ldots, x_{p}\right)=0$ and the system $T$ have the same number of solutions in $\boldsymbol{K}$.

Lemma 2. ([8, p. 100]) If $\boldsymbol{L} \in \mathcal{R} \cup\{\mathbb{N}, \mathbb{N} \backslash\{0\}\}$ and $x, y, z \in \boldsymbol{L}$, then $z(x+y-z)=0$ if and only if

$$
(z x+1)(z y+1)=z^{2}(x y+1)+1
$$

Let $\alpha, \beta$, and $\gamma$ denote variables.

Lemma 3. If $\boldsymbol{L} \in \mathcal{R} \cup\{\mathbb{N}, \mathbb{N} \backslash\{0\}\}$ and $x, y, z \in \boldsymbol{L}$, then $x+y=z$ if and only if

$$
(z x+1)(z y+1)=z^{2}(x y+1)+1
$$

and

$$
((z+1) x+1)((z+1)(y+1)+1)=(z+1)^{2}(x(y+1)+1)+1
$$

We can express equations (1) and (2) as a system $\mathcal{F}$ such that $\mathcal{F}$ involves $x, y, z$ and 20 new variables and $\mathcal{F}$ consists of equations of the forms $\alpha+1=\gamma$ and $\alpha \cdot \beta=\gamma$.

Proof. By Lemma 2, equation (1) is equivalent to

$$
z(x+y-z)=0
$$

and equation (2) is equivalent to

$$
(z+1)(x+(y+1)-(z+1))=0
$$

The conjunction of equations (3) and (4) is equivalent to $x+y=z$. The new 20 variables express the following 20 polynomials: 


$$
\begin{gathered}
z x, \quad z x+1, \quad z y, \quad z y+1, \quad z^{2}, \quad x y, \quad x y+1, \\
z^{2}(x y+1), \quad z^{2}(x y+1)+1, \quad z+1, \quad(z+1) x, \quad(z+1)(y+1)+1, \\
(z+1) x+1, \quad y+1, \quad(z+1)(y+1), \quad x(y+1)+1, \\
(z+1)^{2}, \quad x(y+1), \quad(z+1)^{2}(x(y+1)+1)+1 .
\end{gathered}
$$

Lemma 4. (cf. Observation 4) Let $D\left(x_{1}, \ldots, x_{p}\right) \in$ $\mathbb{Z}\left[x_{1}, \ldots, x_{p}\right]$. Assume that $\operatorname{deg}\left(D, x_{i}\right) \geqslant 1$ for each $i \in\{1, \ldots, p\}$. We can compute a positive integer $n>p$ and a system $T \subseteq G_{n}$ which satisfies the following two conditions:

Condition 4. If $\boldsymbol{K} \in \mathcal{R} n g \cup\{\mathbb{N}, \mathbb{N} \backslash\{0\}\}$, then

$$
\begin{gathered}
\forall \tilde{x}_{1}, \ldots, \tilde{x}_{p} \in \boldsymbol{K}\left(D\left(\tilde{x}_{1}, \ldots, \tilde{x}_{p}\right)=0 \Longleftrightarrow\right. \\
\left.\exists \tilde{x}_{p+1}, \ldots, \tilde{x}_{n} \in \boldsymbol{K}\left(\tilde{x}_{1}, \ldots, \tilde{x}_{p}, \tilde{x}_{p+1}, \ldots, \tilde{x}_{n}\right) \text { solves } T\right)
\end{gathered}
$$

Condition 5. If $\boldsymbol{K} \in \mathcal{R} n \mathrm{U} \cup\{\mathbb{N}, \mathbb{N} \backslash\{0\}\}$, then for each $\tilde{x}_{1}, \ldots, \tilde{x}_{p} \in \boldsymbol{K}$ with $D\left(\tilde{x}_{1}, \ldots, \tilde{x}_{p}\right)=0$, there exists a unique tuple $\left(\tilde{x}_{p+1}, \ldots, \tilde{x}_{n}\right) \in \boldsymbol{K}^{n-p}$ such that the tuple $\left(\tilde{x}_{1}, \ldots, \tilde{x}_{p}, \tilde{x}_{p+1}, \ldots, \tilde{x}_{n}\right)$ solves $T$.

Conditions 4 and 5 imply that for each $\boldsymbol{K} \in \mathcal{R} n \cup\{\mathbb{N}, \mathbb{N} \backslash\{0\}\}$, the equation $D\left(x_{1}, \ldots, x_{p}\right)=0$ and the system $T$ have the same number of solutions in $\boldsymbol{K}$.

Proof. Let the system $T \subseteq E_{n}$ be given by Lemma 1 . For every $\boldsymbol{L} \in \mathcal{R} \cup\{\mathbb{N}, \mathbb{N} \backslash\{0\}\}$,

$$
\forall x \in \boldsymbol{L}(x=1 \Longleftrightarrow(x \cdot x=x \wedge x \cdot(x+1)=x+1))
$$

Therefore, if there exists $m \in\{1, \ldots, n\}$ such that the equation $1=x_{m}$ belongs to $T$, then we introduce a new variable $y$ and replace in $T$ each equation of the form $1=x_{k}$ by the equations $x_{k} \cdot x_{k}=x_{k}, x_{k}+1=y, x_{k} \cdot y=y$. Next, we apply Lemma 3 to each equation of the form $x_{i}+x_{j}=x_{k}$ that belongs to $T$ and replace in $T$ each such equation by an equivalent system of equations of the forms $\alpha+1=\gamma$ and $\alpha \cdot \beta=\gamma$.

\section{The main consequence of Conjecture 1}

Theorem 2. Conjecture 1 implies that there is an algorithm which takes as input a Diophantine equation, returns an integer, and this integer is greater than the heights of rational solutions, if the solution set is finite.

Proof. It follows from Lemma 4 for $K=\mathbb{Q}$. The claim of Theorem 2 also follows from Observation 4.

Corollary 2. Conjecture 1 implies that the set of all Diophantine equations which have infinitely many rational solutions is recursively enumerable. Assuming Conjecture 1, a single query to the halting oracle decides whether or not a given Diophantine equation has infinitely many rational solutions. By the Davis-Putnam-Robinson-Matiyasevich theorem, the same is true for an oracle that decides whether or not a given Diophantine equation has an integer solution.
For many Diophantine equations we know that the number of rational solutions is finite by Faltings' theorem. Faltings' theorem tells that certain curves have finitely many rational points, but no known proof gives any bound on the sizes of the numerators and denominators of the coordinates of those points, see [5, p. 722]. In all such cases Conjecture 1 allows us to compute such a bound. If this bound is small enough, that allows us to find all rational solutions by an exhaustive search. For example, the equation $x_{1}^{5}-x_{1}=x_{2}^{2}-x_{2}$ has only finitely many rational solutions ([7, p. 212]). The known rational solutions are: $(-1,0),(-1,1),(0,0),(0,1),(1,0),(1,1),(2,-5)$, $(2,6),(3,-15),(3,16),(30,-4929),(30,4930),\left(\frac{1}{4}, \frac{15}{32}\right),\left(\frac{1}{4}, \frac{17}{32}\right)$, $\left(-\frac{15}{16},-\frac{185}{1024}\right),\left(-\frac{15}{16}, \frac{1209}{1024}\right)$, and the existence of other solutions is an open question, see [10, pp. 223-224]. The system

$$
\left\{\begin{array}{l}
x_{3}+1=x_{2} \\
x_{2} \cdot x_{3}=x_{4} \\
x_{5}+1=x_{1} \\
x_{1} \cdot x_{1}=x_{6} \\
x_{6} \cdot x_{6}=x_{7} \\
x_{7} \cdot x_{5}=x_{4}
\end{array}\right.
$$

is equivalent to $x_{1}^{5}-x_{1}=x_{2}^{2}-x_{2}$. By Conjecture $1, h\left(x_{1}^{4}\right)=$ $h\left(x_{7}\right) \leqslant h\left(x_{1}, \ldots, x_{7}\right) \leqslant 2^{2^{7-2}}=2^{32}$. Therefore, $h\left(x_{1}\right) \leqslant$ $\left(2^{32}\right)^{\frac{1}{4}}=256$. Assuming that Conjecture 1 holds, the following MUPAD code finds all rational solutions of the equation $x_{1}^{5}-x_{1}=x_{2}^{2}-x_{2}$.

solutions: $=\{\}$ :

for $i$ from -256 to 256 do

for $j$ from 1 to 256 do

$\mathrm{x}:=\mathrm{i} / \mathrm{j}:$

$y:=4 * x^{\wedge} 5-4 * x+1:$

$\mathrm{p}:=$ numer $(\mathrm{y})$ :

$\mathrm{q}:=\operatorname{denom}(\mathrm{y}):$

if numlib::issqr(p) and numlib::issqr(q) then

$\mathrm{z} 1:=\operatorname{sqrt}(\mathrm{p} / \mathrm{q})$ :

$\mathrm{z} 2:=-\operatorname{sqrt}(\mathrm{p} / \mathrm{q})$

$\mathrm{y} 1:=(\mathrm{z} 1+1) / 2:$

$\mathrm{y} 2:=(\mathrm{z} 2+1) / 2:$

solutions:=solutions union $\{[\mathrm{x}, \mathrm{y} 1],[\mathrm{x}, \mathrm{y} 2]\}$ :

end_if:

end_for:

end_for:

print(solutions):

The code solves the equivalent equation

$$
4 x_{1}^{5}-4 x_{1}+1=\left(2 x_{2}-1\right)^{2}
$$

and displays the already presented solutions.

$M U P A D$ is a general-purpose computer algebra system. The commercial version of MuPAD is no longer available as a stand-alone product, but only as the Symbolic Math Toolbox of MATLAB. Fortunately, this and the next code can be executed by MuPAD Light, which was offered for free for research and education until autumn 2005.

\section{Algebraic lemmas - part 2}

Lemma 5. Lemmas 2 and 3 are not necessary for proving that in the rational domain each Diophantine equation is 
equivalent to a system of equations of the forms $\alpha+1=\gamma$ and $\alpha \cdot \beta=\gamma$.

Proof. By Lemma 1, an arbitrary Diophantine equation is equivalent to a system $T \subseteq E_{n}$, where $n$ and $T$ can be computed. If there exists $m \in\{1, \ldots, n\}$ such that the equation $1=x_{m}$ belongs to $T$, then we introduce a new variable $t$ and replace in $T$ each equation of the form $1=x_{k}$ by the equations $x_{k} \cdot x_{k}=x_{k}, x_{k}+1=t$, and $x_{k} \cdot t=t$. For each rational number $y$, we have $y^{2}+1 \neq 0$ and $y\left(y^{2}+1\right)+1 \neq 0$. Hence, for each rational numbers $x, y, z$,

$$
\begin{gathered}
x+y=z \Longleftrightarrow x\left(y^{2}+1\right)+y\left(y^{2}+1\right)=z\left(y^{2}+1\right) \Longleftrightarrow \\
x\left(y^{2}+1\right)+y\left(y^{2}+1\right)+1=z\left(y^{2}+1\right)+1 \Longleftrightarrow \\
\left(y\left(y^{2}+1\right)+1\right) \cdot\left(\frac{x\left(y^{2}+1\right)}{y\left(y^{2}+1\right)+1}+1\right)=z\left(y^{2}+1\right)+1
\end{gathered}
$$

We transform the last equation into an equivalent system $W \subseteq G_{12}$ in such a way that the variables $x_{1}, \ldots, x_{12}$ correspond to the following rational expressions:

$$
\begin{aligned}
& x, y, z, y^{2}, y^{2}+1, y\left(y^{2}+1\right), y\left(y^{2}+1\right)+1, x\left(y^{2}+1\right), \\
& \frac{x\left(y^{2}+1\right)}{y\left(y^{2}+1\right)+1}, \frac{x\left(y^{2}+1\right)}{y\left(y^{2}+1\right)+1}+1, z\left(y^{2}+1\right), z\left(y^{2}+1\right)+1 .
\end{aligned}
$$

In this way, we replace in $T$ each equation of the form $x_{i}+x_{j}=x_{k}$ by an equivalent system of equations of the forms $\alpha+1=\gamma$ and $\alpha \cdot \beta=\gamma$.

The next lemma enable us to prove Theorem 2 without using Lemma 4.

Lemma 6. For solutions in a field, each system $\mathcal{S} \subseteq E_{n}$ is equivalent to $T_{1} \vee \cdots \vee T_{p}$, where each $T_{i}$ is a system of equations of the forms $\alpha+1=\gamma$ and $\alpha \cdot \beta=\gamma$.

Proof. Acting as in the proof of Lemma 5, we eliminate from $\mathcal{S}$ all equations of the form $1=x_{k}$. Let $m$ denote the number of equations of the form $x_{i}+x_{j}=x_{k}$ that belong to $\mathcal{S}$. We can assume that $m>0$. Let the variables $y, z, t, w, s$, and $r$ be new. Let

$$
\begin{gathered}
\mathcal{S}_{1}=\left(\mathcal{S} \backslash\left\{x_{i}+x_{j}=x_{k}\right\}\right) \cup \\
\left\{x_{i}+1=y, \quad x_{k}+1=y, \quad x_{j}+1=z, \quad z \cdot x_{j}=x_{j}\right\}
\end{gathered}
$$

and let

$$
\begin{gathered}
\mathcal{S}_{2}=\left(\mathcal{S} \backslash\left\{x_{i}+x_{j}=x_{k}\right\}\right) \cup \\
\left\{t \cdot x_{j}=x_{i}, \quad t+1=w, \quad w \cdot x_{j}=x_{k}, \quad x_{j}+1=s, \quad r \cdot x_{j}=s\right\}
\end{gathered}
$$

The system $\mathcal{S}_{1}$ expresses that $x_{i}+x_{j}=x_{k}$ and $x_{j}=0$. The system $\mathcal{S}_{2}$ expresses that $x_{i}+x_{j}=x_{k}$ and $x_{j} \neq 0$. Therefore, $\mathcal{S} \Longleftrightarrow\left(\mathcal{S}_{1} \vee \mathcal{S}_{2}\right)$. We have described a procedure which transforms $\mathcal{S}$ into $\mathcal{S}_{1}$ and $\mathcal{S}_{2}$. We iterate this procedure for $\mathcal{S}_{1}$ and $\mathcal{S}_{2}$ and finally obtain the systems $T_{1}, \ldots, T_{2^{m}}$ without equations of the form $x_{i}+x_{j}=x_{k}$. The systems $T_{1}, \ldots, T_{2^{m}}$ satisfy $\mathcal{S} \Longleftrightarrow\left(T_{1} \vee \cdots \vee T_{2^{m}}\right)$ and they contain only equations of the forms $\alpha+1=\gamma$ and $\alpha \cdot \beta=\gamma$.

\section{Systems which have infinitely many rational solutions}

Lemma 7. ([9, p. 391]) If 2 has an odd exponent in the prime factorization of a positive integer $n$, then $n$ can be written as the sum of three squares of integers.

Lemma 8. For each positive rational number $z, z$ or $2 z$ can be written as the sum of three squares of rational numbers.

Proof. We find positive integers $p$ and $q$ with $z=\frac{p}{q}$. If 2 has an odd exponent in the prime factorization of $p q$, then by Lemma 7 there exist integers $i_{1}, i_{2}, i_{3}$ such that $p q=i_{1}^{2}+i_{2}^{2}+i_{3}^{2}$. Hence,

$$
z=\left(\frac{i_{1}}{q}\right)^{2}+\left(\frac{i_{2}}{q}\right)^{2}+\left(\frac{i_{3}}{q}\right)^{2}
$$

If 2 has an even exponent in the prime factorization of $p q$, then by Lemma 7 there exist integers $j_{1}, j_{2}, j_{3}$ such that $2 p q=j_{1}^{2}+j_{2}^{2}+j_{3}^{2}$. Hence,

$$
2 z=\left(\frac{j_{1}}{q}\right)^{2}+\left(\frac{j_{2}}{q}\right)^{2}+\left(\frac{j_{3}}{q}\right)^{2}
$$

Lemma 9. A rational number $z$ can be written as the sum of three squares of rational numbers if and only if there exist rational numbers $r, s$, $t$ such that $z=r^{2}\left(s^{2}\left(t^{2}+1\right)+1\right)$.

Proof. Let $H(r, s, t)=r^{2}\left(s^{2}\left(t^{2}+1\right)+1\right)$. Of course,

$$
H(r, s, t)=r^{2}+(r s)^{2}+(r s t)^{2}
$$

We prove that for each rational numbers $a, b, c$ there exist rational numbers $r, s, t$ such that $a^{2}+b^{2}+c^{2}=H(r, s, t)$. Without loss of generality we can assume that $|a| \leqslant|b| \leqslant|c|$. If $b=0$, then $a=0$ and $a^{2}+b^{2}+c^{2}=H(c, 0,0)$. If $b \neq 0$, then $c \neq 0$ and $a^{2}+b^{2}+c^{2}=H\left(c, \frac{b}{c}, \frac{a}{b}\right)$.

Lemma 10. ([1, p. 125]) The equation $x^{3}+y^{3}=4981$ has infinitely many solutions in positive rationals and each such solution $(x, y)$ satisfies $h(x, y)>10^{16 \cdot 10^{6}}$.

Theorem 3. There exists a system $\mathcal{T} \subseteq G_{28}$ such that $\mathcal{T}$ has infinitely many solutions in rationals $x_{1}, \ldots, x_{28}$ and each such solution $\left(x_{1}, \ldots, x_{28}\right)$ has height greater than $2^{2^{27}}$.

Proof. We define:

$$
\Omega=\left\{\rho \in \mathbb{Q} \cap(0, \infty): \exists y \in \mathbb{Q}(\rho \cdot y)^{3}+y^{3}=4981\right\}
$$

Let $\Omega_{1}$ denote the set of all positive rationals $\rho$ such that the system

$$
\left\{\begin{aligned}
(\rho \cdot y)^{3}+y^{3} & =4981 \\
\rho^{3} & =a^{2}+b^{2}+c^{2}
\end{aligned}\right.
$$

is solvable in rationals. Let $\Omega_{2}$ denote the set of all positive rationals $\rho$ such that the system

$$
\left\{\begin{aligned}
(\rho \cdot y)^{3}+y^{3} & =4981 \\
2 \rho^{3} & =a^{2}+b^{2}+c^{2}
\end{aligned}\right.
$$


is solvable in rationals. Lemma 10 implies that the set $\Omega$ is infinite. By Lemma $8, \Omega=\Omega_{1} \cup \Omega_{2}$. Therefore, $\Omega_{1}$ is infinite (Case 1) or $\Omega_{2}$ is infinite (Case 2).

Case 1. In this case the system

$$
\left\{\begin{aligned}
x^{3}+y^{3} & =4981 \\
\frac{x^{3}}{y^{3}} & =a^{2}+b^{2}+c^{2}
\end{aligned}\right.
$$

has infinitely many rational solutions. By this and Lemma 9 , the system

$$
\left\{\begin{aligned}
x^{3}+y^{3} & =4981 \\
\frac{x^{3}}{y^{3}} & =r^{2}\left(s^{2}\left(t^{2}+1\right)+1\right)
\end{aligned}\right.
$$

has infinitely many rational solutions. We transform the above system into an equivalent system $\mathcal{T} \subseteq G_{27}$ in such a way that the variables $x_{1}, \cdots, x_{27}$ correspond to the following rational expressions:

$$
x, y, x^{2}, x^{3}, y^{2}, y^{3}, \frac{x^{3}}{y^{3}}, \frac{x^{3}}{y^{3}}+1,
$$

$1,2,4,16,17,289, \frac{289}{4}, \frac{289}{4}+1,293,4981$,

$t, t^{2}, t^{2}+1, s, s^{2}, s^{2}\left(t^{2}+1\right), s^{2}\left(t^{2}+1\right)+1, r, r^{2}$.

The system $\mathcal{T}$ has infinitely many solutions in rationals $x_{1}, \ldots, x_{27}$. Lemma 10 implies that each rational tuple $\left(x_{1}, \ldots, x_{27}\right)$ that solves $\mathcal{T}$ satisfies

$$
h\left(x_{1}, \ldots, x_{27}\right) \geqslant h\left(x_{1}^{3}, x_{2}^{3}\right)=\left(h\left(x_{1}, x_{2}\right)\right)^{3}>10^{48 \cdot 10^{6}}>2^{2^{27}}
$$

Since $G_{27} \subseteq G_{28}, \mathcal{T} \subseteq G_{28}$ and the proof for Case 1 is complete.

Case 2. In this case the system

$$
\left\{\begin{aligned}
x^{3}+y^{3} & =4981 \\
2 \cdot \frac{x^{3}}{y^{3}} & =a^{2}+b^{2}+c^{2}
\end{aligned}\right.
$$

has infinitely many rational solutions. By this and Lemma 9, the system

$$
\left\{\begin{aligned}
x^{3}+y^{3} & =4981 \\
2 \cdot \frac{x^{3}}{y^{3}} & =r^{2}\left(s^{2}\left(t^{2}+1\right)+1\right)
\end{aligned}\right.
$$

has infinitely many rational solutions. We transform the above system into an equivalent system $\mathcal{T} \subseteq G_{28}$ in such a way that the variables $x_{1}, \ldots, x_{28}$ correspond to the following rational expressions:

$$
x, y, x^{2}, x^{3}, y^{2}, y^{3}, \frac{x^{3}}{y^{3}}, 2 \cdot \frac{x^{3}}{y^{3}}, \frac{x^{3}}{y^{3}}+1,
$$

$1,2,4,16,17,289, \frac{289}{4}, \frac{289}{4}+1,293,4981$,

$t, t^{2}, t^{2}+1, s, s^{2}, s^{2}\left(t^{2}+1\right), s^{2}\left(t^{2}+1\right)+1, r, r^{2}$.

The system $\mathcal{T}$ has infinitely many solutions in rationals $x_{1}, \ldots, x_{28}$. Lemma 10 implies that each rational tuple $\left(x_{1}, \ldots, x_{28}\right)$ that solves $\mathcal{T}$ satisfies

$$
h\left(x_{1}, \ldots, x_{28}\right) \geqslant h\left(x_{1}^{3}, x_{2}^{3}\right)=\left(h\left(x_{1}, x_{2}\right)\right)^{3}>10^{48 \cdot 10^{6}}>2^{2^{27}}
$$

For a positive integer $n$, let $\mu(n)$ denote the smallest positive integer $m$ such that each system $\mathcal{S} \subseteq G_{n}$ solvable in rationals $x_{1}, \ldots, x_{n}$ has a rational solution $\left(x_{1}, \ldots, x_{n}\right)$ whose height is not greater than $m$. Obviously, $\mu(1)=1$. Observation 1 implies that $\mu(n) \geqslant 2^{2^{n-2}}$ for every integer $n \geqslant 2$. Theorem 3 implies that $\mu(28)>2^{2^{27}}$.

Theorem 4. The function $\mu: \mathbb{N} \backslash\{0\} \rightarrow \mathbb{N} \backslash\{0\}$ is computable in the limit.

Proof. Let us agree that the empty tuple has height 0 . For a positive integer $w$ and a tuple

$$
\left(x_{1}, \ldots, x_{n}\right) \in([-w, w] \cap \mathbb{Z})^{n} \backslash\{(\underbrace{w, \ldots, w}_{n \text {-times }})\}
$$

let $\operatorname{succ}\left(\left(x_{1}, \ldots, x_{n}\right), w\right)$ denote the successor of $\left(x_{1}, \ldots, x_{n}\right)$ in the co-lexicographic order on $([-w, w] \cap \mathbb{Z})^{n}$. Flowchart 2 illustrates an infinite-time computation of $\mu(n)$.

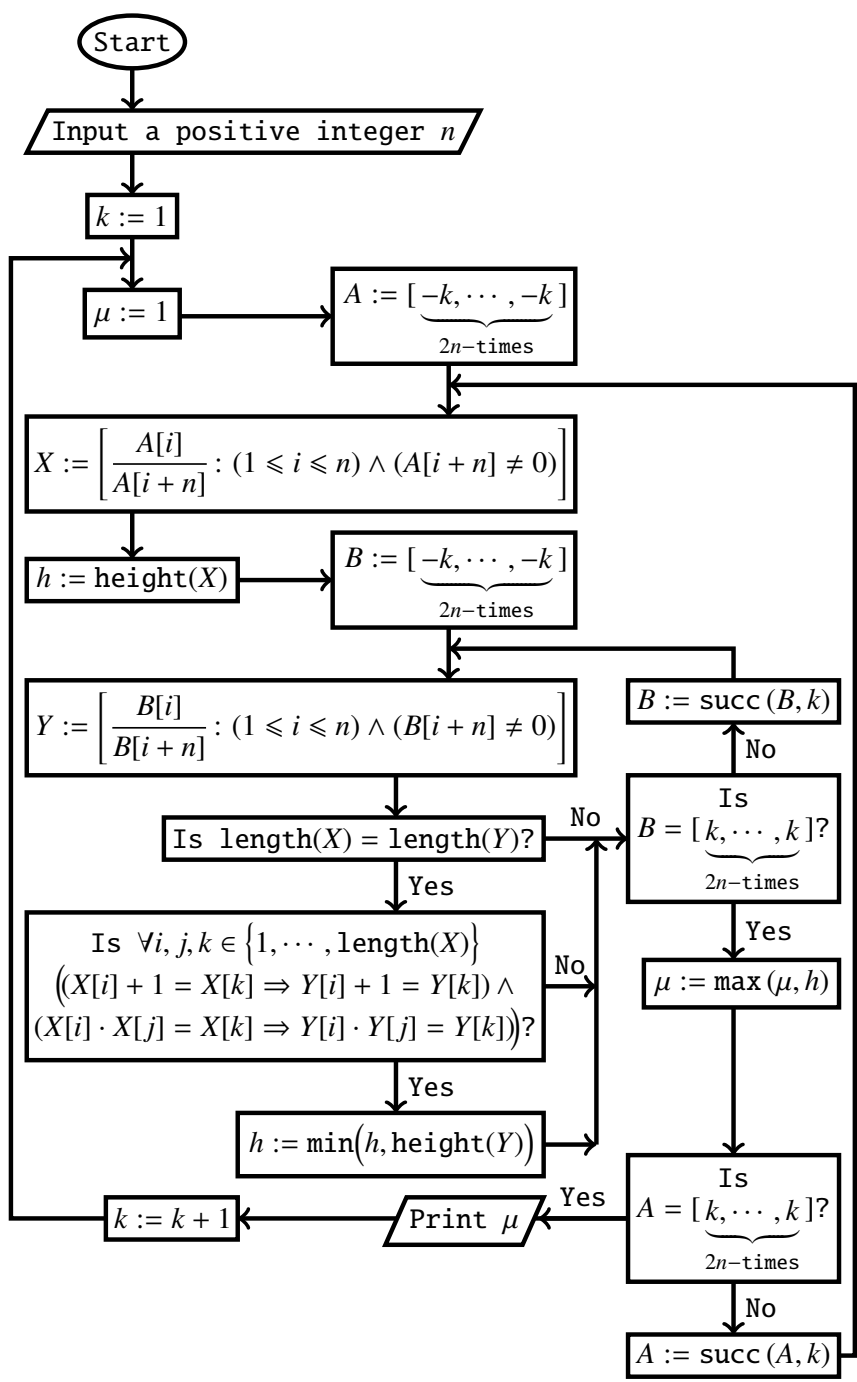

Flowchart 2: An infinite-time computation of $\mu(n)$

The next MuPAD code implements the algorithm from Flowchart 2. In MuPAD, nops(.) denotes the length of a list. 
The code is useless for practical computations because the algorithm from Flowchart 2 is very time-consuming.

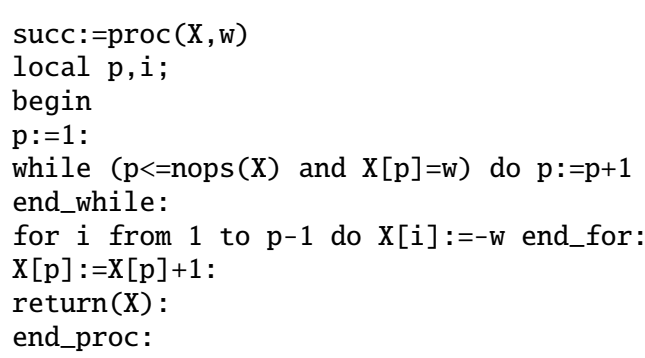

end_for:

$\mathrm{m}:=\max (\mathrm{m}, \mathrm{h})$ :

$\mathrm{X}:=\operatorname{succ}(\mathrm{X}, \mathrm{k}):$

end_for:

print $(\mathrm{m})$ :

$\mathrm{k}:=\mathrm{k}+1$ :

end_while:

\section{Conjecture 2 and its equivalent form}

Let $[\cdot]$ denote the integer part function.

Lemma 11. For every non-negative real numbers $x$ and $y$, $x+1=y$ implies that $2^{2^{[x]}} \cdot 2^{2^{[x]}}=2^{2^{[y]}}$.

Proof. For every non-negative real numbers $x$ and $y, x+1=y$ implies that $[x]+1=[y]$.

Let $f(1)=1$, and let $f(n+1)=2^{2^{f(n)}}$ for every positive integer $n$. Let $g(1)=0$, and let $g(n+1)=2^{2^{g(n)}}$ for every positive integer $n$.

Conjecture 2. If a system $\mathcal{S} \subseteq G_{n}$ has only finitely many solutions in non-negative rationals $x_{1}, \ldots, x_{n}$, then each such solution $\left(x_{1}, \ldots, x_{n}\right)$ satisfies $h\left(x_{1}, \ldots, x_{n}\right) \leqslant f(2 n)$.

Observations 2 and 3 justify Conjecture 2 .

Observation 2. For every system $\mathcal{S} \subseteq G_{n}$ which involves all the variables $x_{1}, \ldots, x_{n}$, the following new system

$$
\mathcal{S} \cup\left\{2^{2^{\left[x_{k}\right]}}=y_{k}: k \in\{1, \ldots, n\}\right\} \cup \bigcup_{x_{i}+1=x_{k} \in \mathcal{S}}\left\{y_{i} \cdot y_{i}=y_{k}\right\}
$$

is equivalent to $\mathcal{S}$. If the system $\mathcal{S}$ has only finitely many solutions in non-negative rationals $x_{1}, \ldots, x_{n}$, then the new system has only finitely many solutions in non-negative rationals $x_{1}, \ldots, x_{n}, y_{1}, \ldots, y_{n}$

Proof. It follows from Lemma 11.

Observation 3. For every positive integer n, the following system

$$
\left\{\begin{aligned}
x_{1} \cdot x_{1} & =x_{1} \\
\forall i \in\{1, \ldots, n-1\} 2^{2^{\left[x_{i}\right]}} & \left.=x_{i+1} \text { (if } n>1\right)
\end{aligned}\right.
$$

has exactly two solutions in non-negative rationals, namely $(g(1), \ldots, g(n))$ and $(f(1), \ldots, f(n))$. The second solution has greater height.

Conjecture 2 is equivalent to the following conjecture on rational arithmetic: if non-negative rational numbers $x_{1}, \ldots, x_{n}$ satisfy $h\left(x_{1}, \ldots, x_{n}\right)>f(2 n)$, then there exist non-negative rational numbers $y_{1}, \ldots, y_{n}$ such that

$$
h\left(x_{1}, \ldots, x_{n}\right)<h\left(y_{1}, \ldots, y_{n}\right)
$$

and for every $i, j, k \in\{1, \ldots, n\}$

$$
\left(x_{i}+1=x_{k} \Longrightarrow y_{i}+1=y_{k}\right) \wedge\left(x_{i} \cdot x_{j}=x_{k} \Longrightarrow y_{i} \cdot y_{j}=y_{k}\right)
$$

Theorem 5. Conjecture 2 is true if and only if the execution of Flowchart 3 prints infinitely many numbers. 


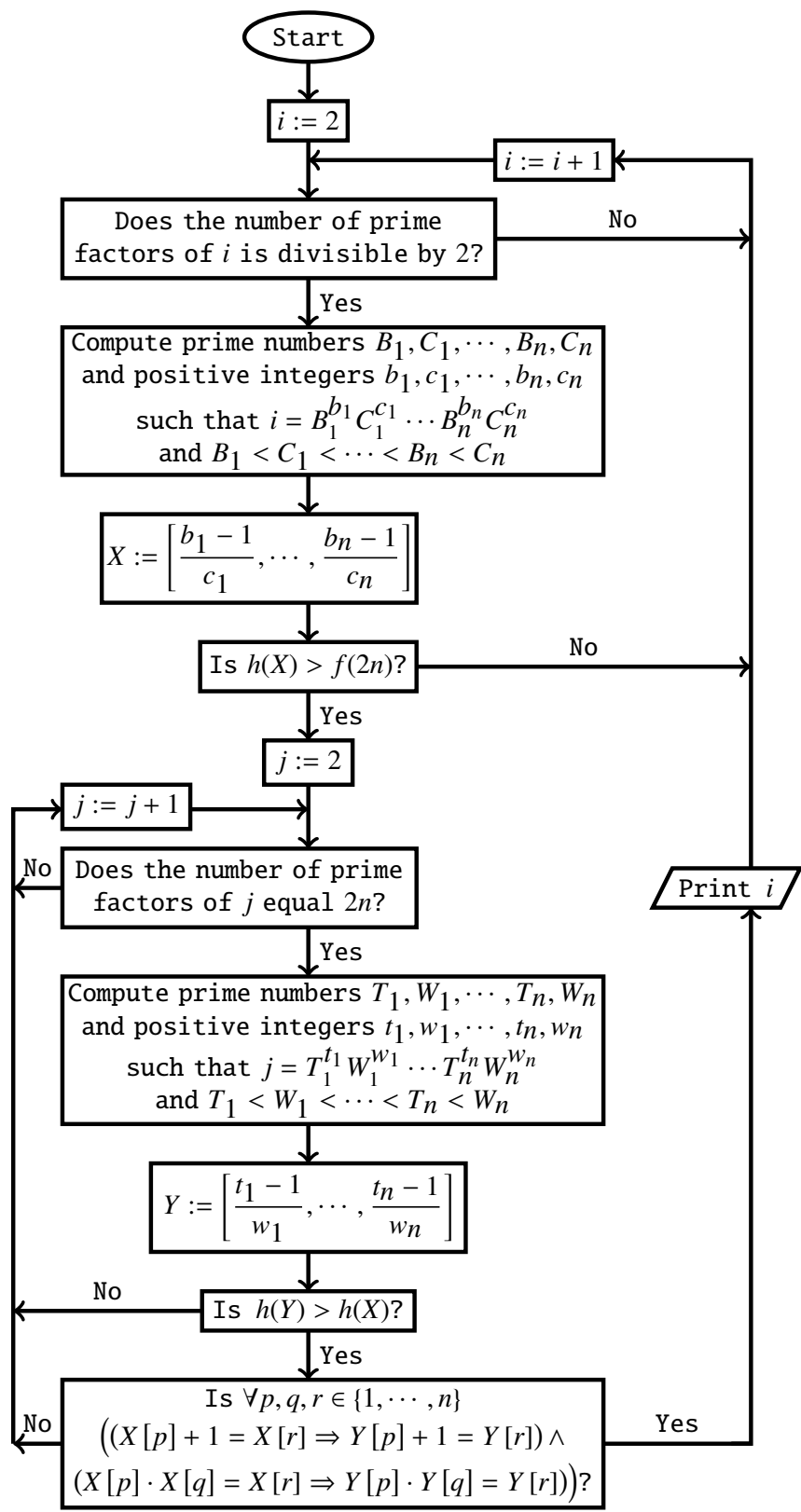

Flowchart 3: An infinite-time computation which decides whether or not Conjecture 2 is true

Proof. Let $\Gamma_{2}$ denote the set of all integers $i \geqslant 2$ whose number of prime factors is divisible by 2 . The claimed equivalence is true because the algorithm from Flowchart 3 applies a surjective function from $\Gamma_{2}$ to $\bigcup_{n=1}^{\infty}(\mathbb{Q} \cap[0, \infty))^{n}$.

Corollary 3. Conjecture 2 can be written in the form $\forall x \in \mathbb{N} \exists y \in \mathbb{N} \psi(x, y)$, where $\psi(x, y)$ is a computable predicate.

\section{Algebraic lemmas - part 3}

Lemma 12. (cf. [8, p. 100]) For every non-negative real numbers $x, y, z, x+y=z$ if and only if

$$
((z+1) x+1)((z+1)(y+1)+1)=(z+1)^{2}(x(y+1)+1)+1
$$

Proof. The left side of equation (5) minus the right side of equation (5) equals $(z+1)(x+y-z)$.

Lemma 13. In non-negative rationals, the equation $x+y=z$ is equivalent to a system which consists of equations of the forms $\alpha+1=\gamma$ and $\alpha \cdot \beta=\gamma$.

Proof. It follows from Lemma 12.

Lemma 14. Let $D\left(x_{1}, \ldots, x_{p}\right) \in \mathbb{Z}\left[x_{1}, \ldots, x_{p}\right]$. Assume that $\operatorname{deg}\left(D, x_{i}\right) \geqslant 1$ for each $i \in\{1, \ldots, p\}$. We can compute a positive integer $n>p$ and a system $\mathcal{T} \subseteq G_{n}$ which satisfies the following two conditions:

Condition 6 . For every non-negative rationals $\tilde{x}_{1}, \ldots, \tilde{x}_{p}$,

$$
D\left(\tilde{x}_{1}, \ldots, \tilde{x}_{p}\right)=0 \Longleftrightarrow
$$

$\exists \tilde{x}_{p+1}, \ldots, \tilde{x}_{n} \in \mathbb{Q} \cap[0, \infty)\left(\tilde{x}_{1}, \ldots, \tilde{x}_{p}, \tilde{x}_{p+1}, \ldots, \tilde{x}_{n}\right)$ solves $\mathcal{T}$

Condition 7. If non-negative rationals $\tilde{x}_{1}, \ldots, \tilde{x}_{p}$ satisfy $D\left(\tilde{x}_{1}, \ldots, \tilde{x}_{p}\right)=0$, then there exists a unique tuple $\left(\tilde{x}_{p+1}, \ldots, \tilde{x}_{n}\right) \in(\mathbb{Q} \cap[0, \infty))^{n-p}$ such that the tuple $\left(\tilde{x}_{1}, \ldots, \tilde{x}_{p}, \tilde{x}_{p+1}, \ldots, \tilde{x}_{n}\right)$ solves $\mathcal{T}$.

Conditions 6 and 7 imply that the equation $D\left(x_{1}, \ldots, x_{p}\right)=0$ and the system $\mathcal{T}$ have the same number of solutions in non-negative rationals.

Proof. We write down the polynomial $D\left(x_{1}, \ldots, x_{p}\right)$ and replace each coefficient by the successor of its absolute value. Let $\widetilde{D}\left(x_{1}, \ldots, x_{p}\right)$ denote the obtained polynomial. The polynomials $D\left(x_{1}, \ldots, x_{p}\right)+\widetilde{D}\left(x_{1}, \ldots, x_{p}\right)$ and $\widetilde{D}\left(x_{1}, \ldots, x_{p}\right)$ have positive integer coefficients. The equation $D\left(x_{1}, \ldots, x_{p}\right)=0$ is equivalent to

$$
D\left(x_{1}, \ldots, x_{p}\right)+\widetilde{D}\left(x_{1}, \ldots, x_{p}\right)+1=\widetilde{D}\left(x_{1}, \ldots, x_{p}\right)+1
$$

There exist a positive integer $a$ and a finite non-empty list $A$ such that

$$
\begin{gathered}
D\left(x_{1}, \ldots, x_{p}\right)+\widetilde{D}\left(x_{1}, \ldots, x_{p}\right)+1= \\
(\left(\sum_{\left(i_{1}, j_{1}, \ldots, i_{k}, j_{k}\right) \in A} x_{i_{1}}^{j_{1}} \cdot \ldots \cdot x_{i_{k}}^{j_{k}}\right)+\underbrace{1)+\ldots)+1}_{a \text { units }}
\end{gathered}
$$

and all the numbers $k, i_{1}, j_{1}, \ldots, i_{k}, j_{k}$ belong to $\mathbb{N} \backslash\{0\}$. There exist a positive integer $b$ and a finite non-empty list $B$ such that

$$
\begin{aligned}
& \widetilde{D}\left(x_{1}, \ldots, x_{p}\right)+1= \\
& \left((\left(\sum_{\left.i_{1}, j_{1}, \ldots, i_{k}, j_{k}\right) \in B} x_{i_{1}}^{j_{1}} \cdot \ldots \cdot x_{i_{k}}^{j_{k}}\right)+\underbrace{1)+\ldots)+1}_{b \text { units }}\right.
\end{aligned}
$$

and all the numbers $k, i_{1}, j_{1}, \ldots, i_{k}, j_{k}$ belong to $\mathbb{N} \backslash\{0\}$. By Lemma 13, we can equivalently express the equality of the right sides of equations (6) and (7) using only equations of the forms $\alpha+1=\gamma$ and $\alpha \cdot \beta=\gamma$. Consequently, we can effectively find the system $\mathcal{T}$.

Observation 4. Combining the above reasoning with Lemma 3 for $\boldsymbol{L}=\mathbb{Q}$, we can prove Lemma 4 for $\boldsymbol{K}=\mathbb{Q}$. 


\section{Consequences of Conjecture 2}

Theorem 6. If we assume Conjecture 2 and a Diophantine equation $D\left(x_{1}, \ldots, x_{p}\right)=0$ has only finitely many solutions in non-negative rationals, then an upper bound for their heights can be computed.

Proof. It follows from Lemma 14.

Theorem 7. If we assume Conjecture 2 and a Diophantine equation $D\left(x_{1}, \ldots, x_{p}\right)=0$ has only finitely many rational solutions, then an upper bound for their heights can be computed by applying Theorem 6 to the equation

$$
\prod_{\left(i_{1}, \ldots, i_{p}\right) \in\{1,2\}^{p}} D\left((-1)^{i_{1}} \cdot x_{1}, \ldots,(-1)^{i_{p}} \cdot x_{p}\right)=0
$$

Corollary 4. Conjecture 2 implies that the set of all Diophantine equations which have infinitely many rational solutions is recursively enumerable. Assuming Conjecture 2, a single query to the halting oracle decides whether or not a given Diophantine equation has infinitely many rational solutions. By the Davis-Putnam-Robinson-Matiyasevich theorem, the same is true for an oracle that decides whether or not a given Diophantine equation has an integer solution.

\section{$X$. Theorems on relative decidability}

Question ([3]). Can the twin prime problem be solved with a single use of a halting oracle?

Let $\xi(3)=4$, and let $\xi(n+1)=\xi(n)$ ! for every integer $n \geqslant 3$. For an integer $n \geqslant 3$, let $\Psi_{n}$ denote the statement: if a system $\mathcal{S} \subseteq\left\{x_{i} !=x_{i+1}: 1 \leqslant i \leqslant n-1\right\} \cup$ $\left\{x_{i} \cdot x_{j}=x_{j+1}: 1 \leqslant i \leqslant j \leqslant n-1\right\}$ has only finitely many solutions in positive integers $x_{1}, \ldots, x_{n}$, then each such solution $\left(x_{1}, \ldots, x_{n}\right)$ satisfies $x_{1}, \ldots, x_{n} \leqslant \xi(n)$.

Theorem 8. ([13]) The statement $\Psi_{16}$ proves the implication: if there exists a twin prime greater than $\xi(14)$, then there are infinitely many twin primes.

Corollary 5. Assuming the statement $\Psi_{16}$, a single query to the halting oracle decides the validity of the twin prime conjecture.

Conjecture 3. Harvey Friedman's conjecture in [4]: the set of all Diophantine equations which have only finitely many rational solutions is not recursively enumerable.

Conjecture 3 implies Conjecture 4.

Conjecture 4. The set of all Diophantine equations which have only finitely many rational solutions is not computable.

By Theorem 2, Conjecture 1 implies Conjecture 5. By Theorem 7, Conjecture 2 implies Conjecture 5.

Conjecture 5. There is an algorithm which takes as input a Diophantine equation $D\left(x_{1}, \ldots, x_{p}\right)=0$, returns an integer $b \geqslant 2$, where $b$ is greater than the number of rational solutions, if the solution set is finite.
Guess ([6, p. 16]). The question whether or not a given Diophantine equation has only finitely many rational solutions is decidable with an oracle that decides whether or not a given Diophantine equation has a rational solution.

Originally, Minhyong Kim formulated the Guess as follows: for rational solutions, the finiteness problem is decidable relative to the existence problem. Conjecture 4 and the Guess imply that there is no algorithm which decides whether or not a Diophantine equation has a rational solution. Martin Davis' conjecture in [2, p. 729] implies the same.

Theorem 9. Conjecture 5 implies that the question whether or not a given Diophantine equation has only finitely many rational solutions is decidable by a single query to an oracle that decides whether or not a given Diophantine equation has a rational solution.

Proof. Assuming that Conjecture 5 holds, the execution of Flowchart 4 decides whether or not a Diophantine equation $D\left(x_{1}, \ldots, x_{p}\right)=0$ has only finitely many rational solutions.

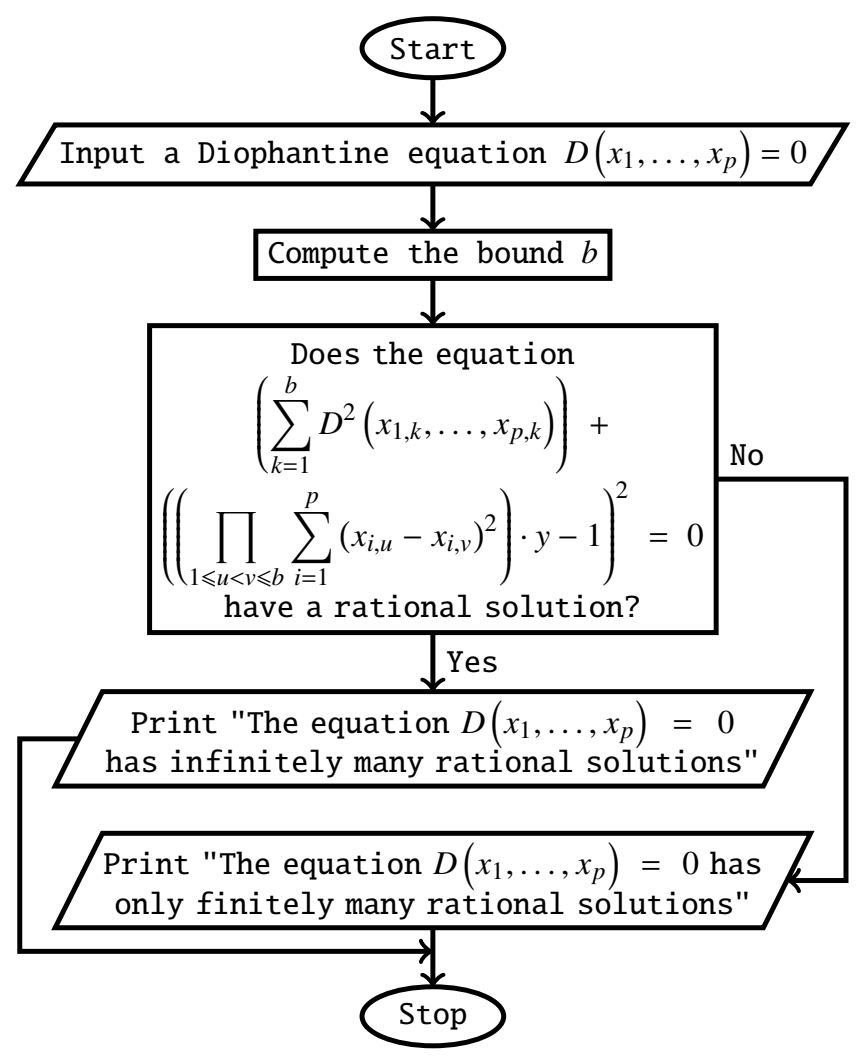

Flowchart 4: Conjecture 5 implies the Guess

Corollary 6. Conjecture 5 implies that the question whether or not a given Diophantine equation has only finitely many rational solutions is decidable by a single query to an oracle that decides whether or not a given Diophantine equation has an integer solution. 
Lemma 15. A Diophantine equation $D\left(x_{1}, \ldots, x_{p}\right)=0$ has no solutions in rationals (alternatively, non-negative integers) $x_{1}, \ldots, x_{p}$ if and only if the equation $D\left(x_{1}, \ldots, x_{p}\right)+0 \cdot x_{p+1}=0$ has only finitely many solutions in rationals (respectively, non-negative integers) $x_{1}, \ldots, x_{p+1}$.

Theorem 10. If the set of all Diophantine equations which have only finitely many rational solutions is recursively enumerable, then there exists an algorithm which decides whether or not a Diophantine equation has a rational solution.

Proof. For a non-negative integer $n$, we define

$$
\theta(n)= \begin{cases}\eta(n+2) & \left(\text { if } n+2 \in \Gamma_{3}\right) \\ 0 & \left(\text { if } n+2 \notin \Gamma_{3}\right)\end{cases}
$$

where $\eta$ and $\Gamma_{3}$ were defined in the proof of Theorem 1. The function $\theta: \mathbb{N} \rightarrow \bigcup_{n=1}^{\infty} \mathbb{Q}^{n}$ is computable and surjective. Suppose that $\left\{\mathcal{A}_{n}=0\right\}_{n=0}^{\infty}$ is a computable sequence of all Diophantine equations which have only finitely many rational solutions. By Lemma 15, the execution of Flowchart 5 decides whether or not a Diophantine equation $D\left(x_{1}, \ldots, x_{p}\right)=0$ has a rational solution.

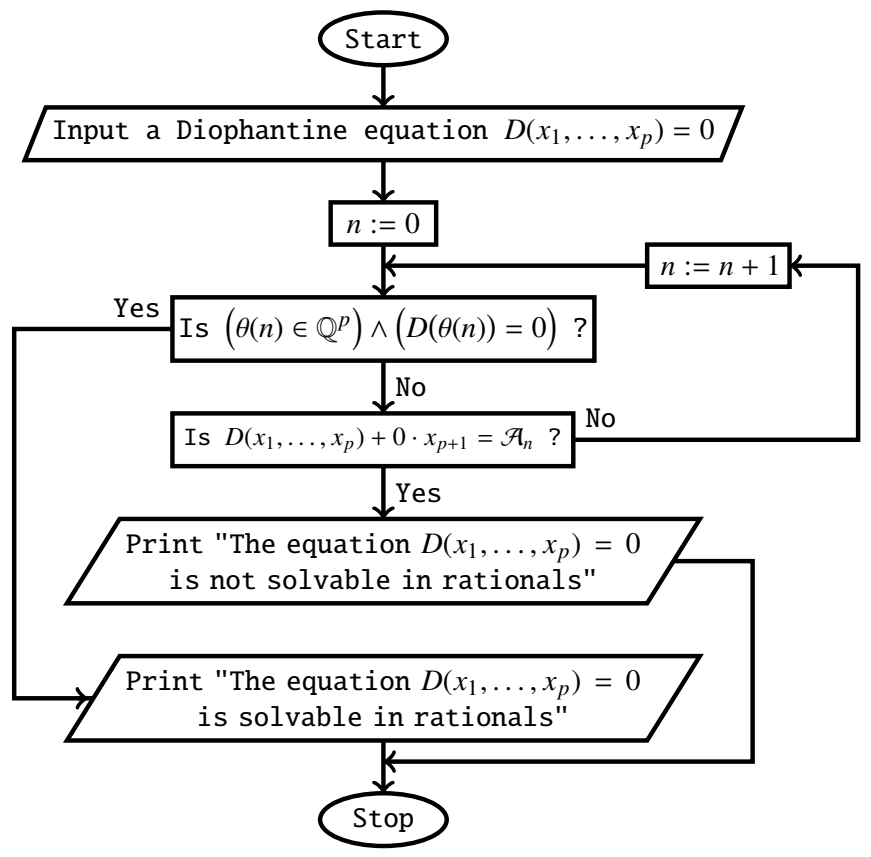

Flowchart 5: An algorithm that decides the solvability of a Diophantine equation $D\left(x_{1}, \ldots, x_{p}\right)=0$ in rationals, if the set of all Diophantine equations which have at most finitely many rational solutions is recursively enumerable

Acknowledgement. Apoloniusz Tyszka wrote the mathematical part of the article. The other authors prepared computer programs in MuPAD.

\section{Summary of the MaIn theorems AND CONJECTURES}

Flowchart 6 provides an overview of the main theorems and conjectures.

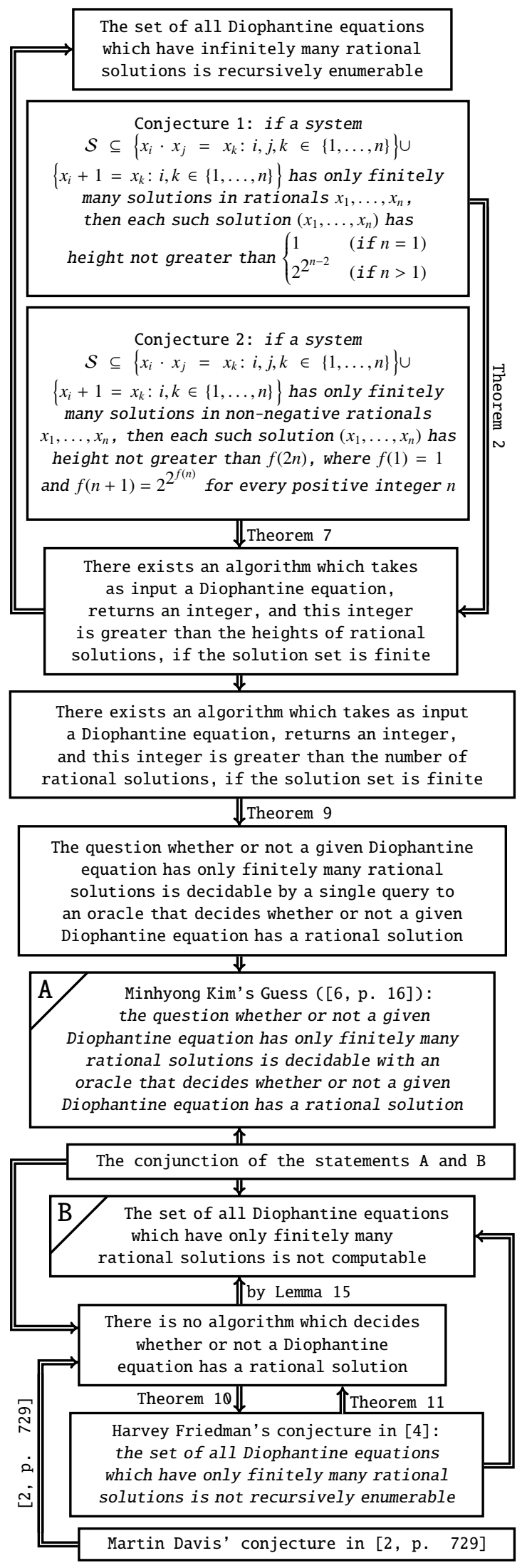

Flowchart 6: Implications between conjectures 


\section{ADDED IN PROOF}

Theorem 11. A positive solution to Hilbert's Tenth Problem for $\mathbb{Q}$ implies that Friedman's conjecture is false.

Proof. Assume a positive solution to Hilbert's Tenth Problem for $\mathbb{Q}$. The algorithm presented in Flowchart 7 stops if and only if a Diophantine equation $D\left(x_{1}, \ldots, x_{p}\right)=0$ has at most finitely many rational solutions.

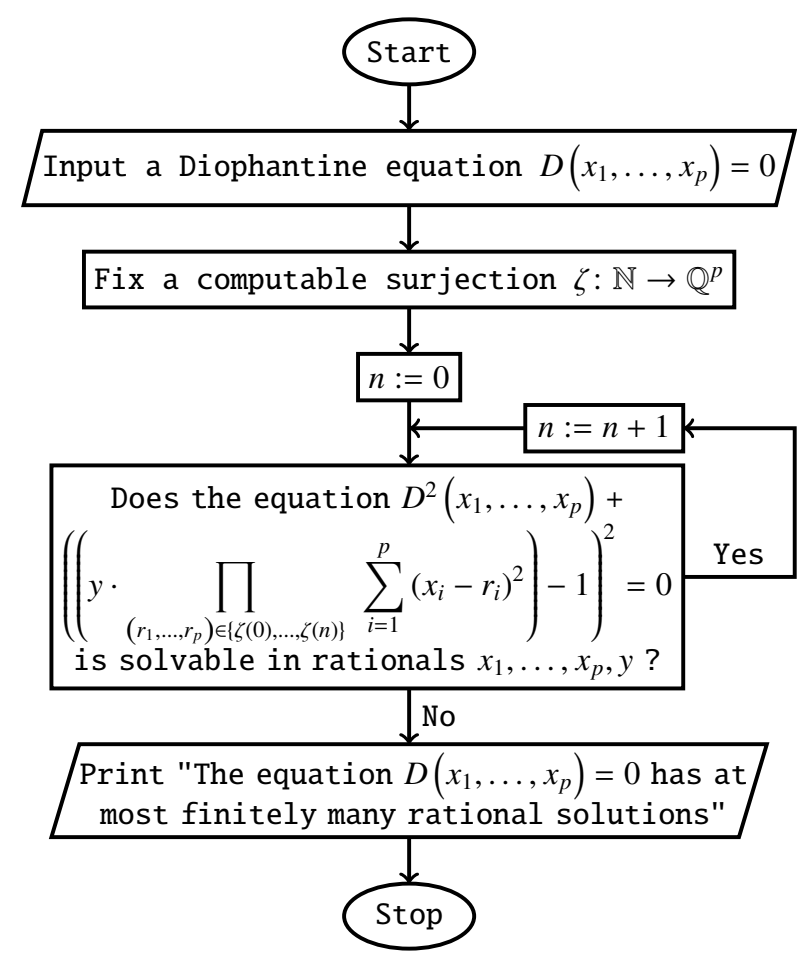

Flowchart 7: A positive solution to Hilbert's Tenth Problem for $\mathbb{Q}$ implies that Friedman's conjecture is false

The set of all Diophantine equations which have at most finitely many solutions in non-negative integers is not recursively enumerable (Smoryński's theorem), see [11, p. 104, Corollary 1].

Theorem 12. If the set of all Diophantine equations which have at most finitely many solutions in non-negative integers is recursively enumerable, then there exists an algorithm which decides whether or not a given Diophantine equation has a solution in non-negative integers. By this and Matiyasevich's theorem, the set of all Diophantine equations which have at most finitely many solutions in non-negative integers is not recursively enumerable.

Proof. Suppose that $\left\{\mathcal{S}_{i}=0\right\}_{i=2}^{\infty}$ is a computable sequence of all Diophantine equations which have at most finitely many solutions in non-negative integers. The algorithm presented in Flowchart 8 uses a computable surjection from $\mathbb{N} \backslash\{0,1\}$ onto $\mathbb{N}^{p}$. By this and Lemma 15, the execution of Flowchart 8 decides whether or not a Diophantine equation $D\left(x_{1}, \ldots, x_{p}\right)=0$ has a solution in non-negative integers.

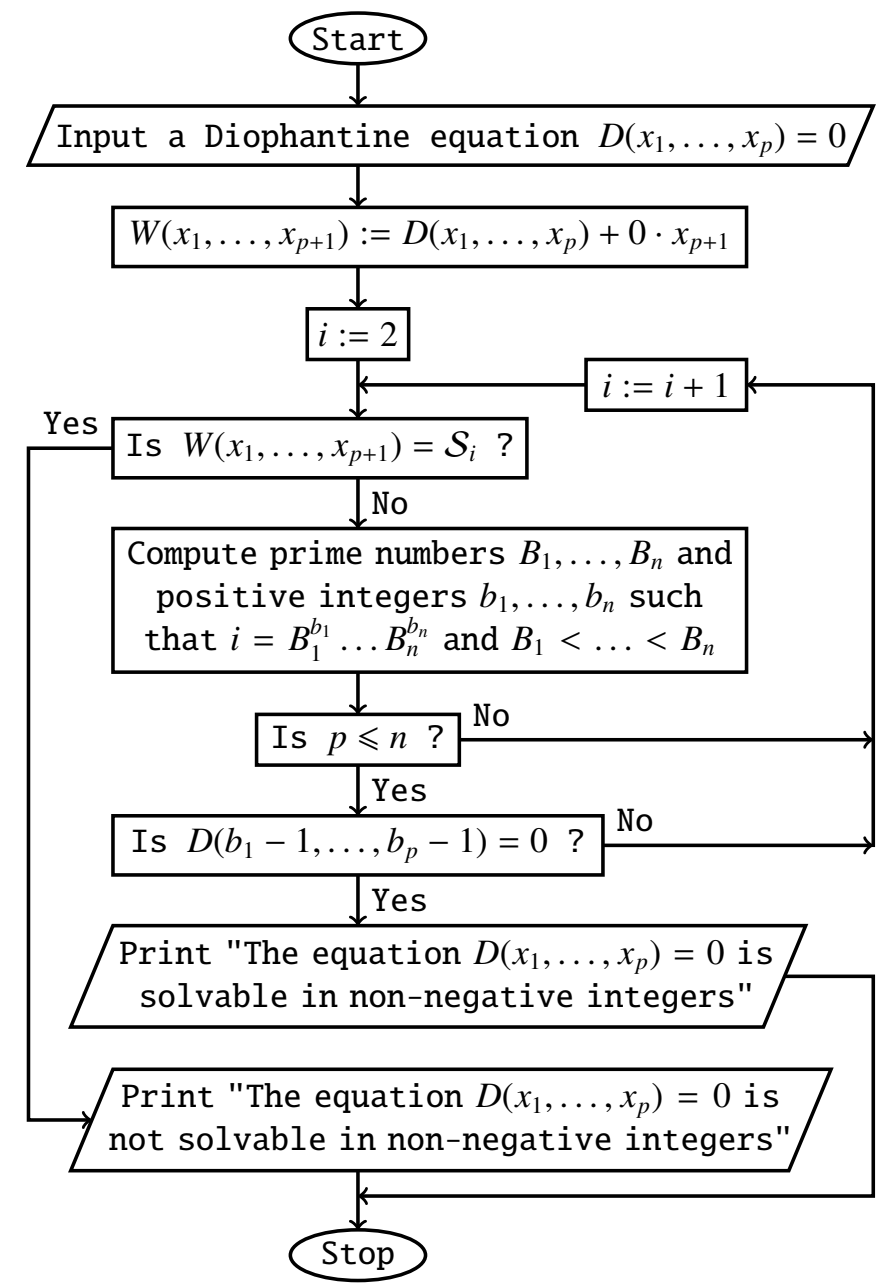

Flowchart 8: A new proof of Smoryński's theorem

\section{REFERENCES}

[1] A. Bremner, Positively prodigious powers or how Dudeney done it? Math. Mag. 84 (2011), no. 2, 120-125, http://dx.doi.org/10.4169/math.mag.84.2.120.

[2] M. Davis, Representation theorems for recursively enumerable sets and a conjecture related to Poonen's large subring of $\mathbb{Q}$, J. Math. Sci. (N. Y.) 171 (2010), no. 6, 728-730. http://dx.doi.org/10.1007/s10958-010-0176-7.

[3] F. G. Dorais, Can the twin prime problem be solved with a single use of a halting oracle? July 23, 2011, http://mathoverflow.net/questions/71050.

[4] H. Friedman, Complexity of statements, April 20, 1998, http://www.cs.nyu.edu/ pipermail/fom/1998-April/001843.html.

[5] T. Gowers, J. Barrow-Green, I. Leader (eds.), The Princeton companion to mathematics, Princeton University Press, Princeton, 2008.

[6] M. Kim, On relative computability for curves, Asia Pac. Math. Newsl. 3 (2013), no. 2, 16-20, http://www.asiapacific-mathnews.com/03/0302/0016 0020.pdf.

[7] M. Mignotte and A. Pethő, On the Diophantine equation $x^{p}-x \overline{=} y^{q}-y$, Publ. Mat. 43 (1999), no. 1, 207-216.

[8] J. Robinson, Definability and decision problems in arithmetic, J. Symbolic Logic 14 (1949), 98-114; reprinted in: The collected works of Julia Robinson (ed. S. Feferman), Amer. Math. Soc. Providence, RI, 1996, 7-23.

[9] W. Sierpiński, Elementary theory of numbers, 2nd ed. (ed. A. Schinzel), PWN (Polish Scientific Publishers) and North-Holland, Warsaw-Amsterdam, 1987.

[10] S. Siksek, Chabauty and the Mordell-Weil Sieve, in: Advances on Superelliptic Curves and Their Applications (eds. L. Beshaj, T. Shaska, E. Zhupa), 194-224, IOS Press, Amsterdam, 2015, http://dx.doi.org/10.3233/978-1-61499-520-3-194.

[11] C. Smoryński, A note on the number of zeros of polynomials and exponential polynomials, J. Symbolic Logic 42 (1977), no. 1, 99-106.

[12] A. Tyszka, Conjecturally computable functions which unconditionally do not have any finite-fold Diophantine representation, Inform. Process. Lett. 113 (2013), no. 19-21, 719-722, http://dx.doi.org/10.1016/j.ipl.2013.07.004.

[13] A. Tyszka, A common approach to Brocard's problem, Landau's problem, and the twin prime problem, March 28, 2017, http://arxiv.org/abs/1506.08655v21. 